\section{Subacute food bolus obstruction secondary to a migrated Overstitch suture from a previous esophageal perforation repair}

The Overstitch device has been used for closure of esophageal perforations [1]. A 40-year-old woman with type I achalasia underwent fluoroscopic pneumatic dilation in September 2015. Her mean lower esophageal sphincter (LES) pressure was $40 \mathrm{mmHg}$. She had $100 \%$ esophageal aperistalsis and absence of LES relaxation. Endoscopy showed a tight but traversable esophagogastric junction (EGJ). A 30-mm

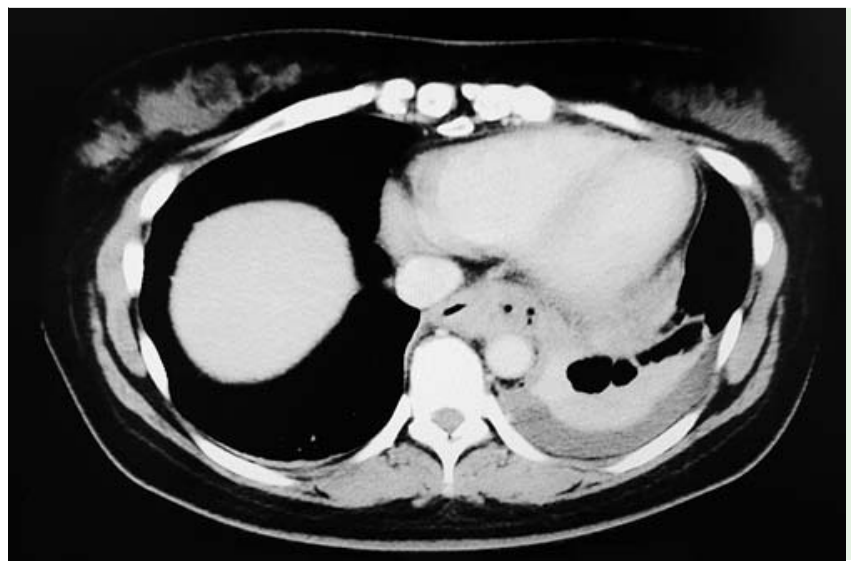

Fig. 1 Computed tomography (CT) scan showing a distal esophageal perforation with extraluminal gas locules abutting the posterior surface of the descending thoracic aorta at the level of the hiatus, along with a small paraesophageal collection and left basal pleural effusion.
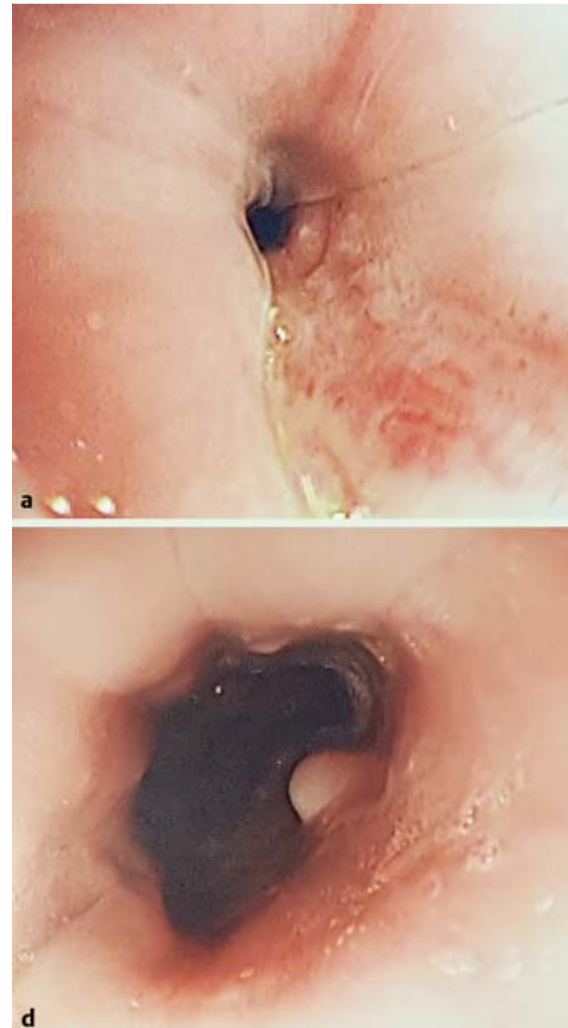
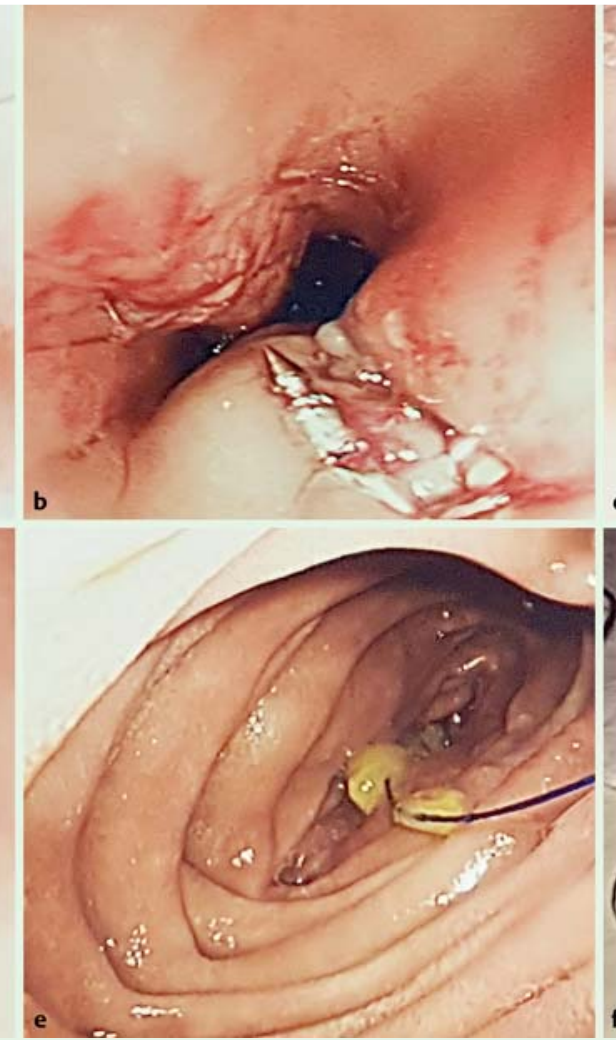

achalasia balloon was used to perform dilation for 1 minute at 5 PSI followed by 1 minute at 8 PSI.

She became pyrexial on the third day after dilation. Computed tomography (CT) showed a distal esophageal perforation with a small paraesophageal collection and left basal pleural effusion ( $\bullet$ Fig. 1 ). Intravenous antibiotics were commenced and ultrasound-guided aspiration of the pleural fluid yielded $8 \mathrm{~mL}$ of hemoserous fluid. An endoscopy on day 6 after dilation showed a 5-mm linear laceration above the EGJ ( $\bullet$ Fig.2a). It was repaired with two Overstitch polydioxanone (PDS) sutures using an Olympus 2T160 gastroscope ( $\bullet$ Fig. 2 b). Endoscopy and fluoroscopy 6 days after this repair showed that the repair was intact and the patient was discharged 13 days after the initial dilation. Gastroscopy at 4 weeks after repair showed good healing of the laceration with residual sutures at the repair site $(\bullet$ Fig. 2 c). The patient's LES pressure was $20.7 \mathrm{mmHg}$; however, she was asymptomatic.

At follow-up at 5 months, the patient complained of spasmodic epigastric pain without vomiting or dysphagia. A subse-

Fig. 2 a-e Endoscopic views showing: a a 5-mm linear deep laceration just above the esophagogastric junction; b repair of the laceration with Overstitch polydioxanone (PDS) sutures; $\mathbf{c}$ good healing of the laceration with a residual suture visible at the repair site 4 weeks after the Overstitch repair; $\mathbf{d}$ complete healing of the laceration and no sutures visible at the repair site 6 months after the Overstitch repair; e the suture that was found lodged at the D2/3 junction during the same endoscopic examination. $\mathbf{f}$ The Overstitch suture along with the food bolus around its $\mathrm{T}$ tag after it had been extracted endoscopically. 
quent endoscopy showed good healing of the perforation site ( $\nabla$ Fig. 2 d); however, a suture with a $3-\mathrm{cm}$ food bolus around its $\mathrm{T}$ tag was lodged at the D2/3 junction ( $\bullet$ Fig. 2e). The suture and the food bolus was completely removed endoscopically using rat-tooth forceps ( $\mathbf{F i g} . \mathbf{2 f}$ ). The patient's symptoms resolved after this procedure.

Endoscopy_UCTN_Code_CPL_1AH_2AJ

Competing interests: None
Baldwin P. M. Yeung, Shannon

M. Chan, Philip W. Y. Chiu

Department of Surgery, Prince of Wales Hospital, The Chinese University of Hong Kong, Hong Kong, China

\section{Reference}

1 Henderson JB, Sorser SA, Atia AN et al. Repair of esophageal perforations using a novel endoscopic suturing system. Gastrointest Endosc 2014; 80: 535-537

\section{Bibliography}

Dol http://dx.doi.org/

10.1055/s-0042-106965

Endoscopy 2016; 48: E177-E178

(c) Georg Thieme Verlag KG

Stuttgart · New York

ISSN 0013-726X

\section{Corresponding author}

\section{Baldwin P. M. Yeung, MBChB, PhD}

Chinese University of Hong Kong - Surgery 30-32 Ngan Shing Street

Shatin

NT Hong Kong

Hong Kong

China

Fax: +852-26377974

byeung@doctors.net.uk 